\title{
Joint Power Control and Beamforming Codebook Design for MISO Channels with Limited Feedback
}

\author{
Behrouz Khoshnevis and Wei Yu \\ Department of Electrical and Computer Engineering \\ University of Toronto, Toronto, Ontario, Canada \\ Email: $\{$ bkhoshnevis, weiyu\}@comm.utoronto.ca
}

\begin{abstract}
This paper investigates the joint design and optimization of the power control and beamforming codebooks for the single-user multiple-input single-output (MISO) wireless systems with a rate-limited feedback link. The problem is cast in the form of minimizing the outage probability subject to the transmit power constraint and cardinality constraints on the beamforming and power codebooks. We show that by appropriately choosing and fixing the beamforming codebook and optimizing the power codebook for that beamforming codebook, it is possible to achieve a performance very close to the optimal joint optimization. Further, this paper investigates the optimal tradeoffs between beamforming and power codebook sizes for different number of feedback bits and transmit antennas. Given a target outage probability, our results provide the optimal codebook sizes independent of the target rate. As the outage probability decreases, we show that the optimal joint design should use fewer feedback bits for beamforming and more feedback bits for power control. The jointly optimized beamforming and power control modules combine the power gain of beamforming and diversity gain of power control, which enable it to approach the performance of the system with perfect channel state information as the feedback link capacity increases to infinity - something that is not possible with beamforming or power control alone.
\end{abstract}

\section{INTRODUCTION}

It is well established that the use of multiple antennas can improve the reliability and capacity of wireless links. Realization of these benefits usually requires the channel state information at the transmitter (CSIT). In practical systems, the receiver typically quantizes the channel information and sends the quantization bits back to the transmitter. The study of such systems, generally referred to as limited feedback systems in the literature, is especially relevant for frequency division duplex (FDD) systems, where the transmitter cannot learn the downlink channel via reciprocity.

Consider a block-fading multiple-input single-output (MISO) channel with channel state information at the receiver (CSIR) and a noiseless delay-free feedback link with the capacity of $B$ bits per fading block. In order to maximize the mutual information in each fading block, the transmitter should use a Gaussian input with its covariance matrix belonging to a codebook of $2^{B}$ matrices. This codebook is shared between the transmitter and the receiver. The receiver measures the channel, chooses the best covariance matrix for the current channel realization, and sends the corresponding matrix index back to the transmitter [1]-[3].

The problem of covariance codebook design is a fairly complex and high-dimensional problem. The problem is further complicated when outage minimization is considered as the performance metric, as the case for real-time traffic. For this reason, the existing literature almost exclusively deals with ergodic capacity, e.g. [2], [3].

This paper studies the feedback design problem under the outage criterion and makes several simplifications to assist the design process. First, we restrict the problem to rankone transmit covariance matrices. This is justified by noting that with perfect CSIT, the optimal transmit covariance matrix is of rank one. Therefore, rank-one matrices are asymptotically optimal as $B \rightarrow \infty$. We further assume a specific codebook structure, where the original codebook is described as the product of a power control codebook and a unit-norm beamforming codebook. This structure, although suboptimal, provides the inherent advantages of the product codes, similar to the shape/gain codes in the quantization theory [4]. These advantages include faster encoding (quantization) and lower storage requirement for the codebooks. Moreover, the separation of the beamforming and power control codebooks allows for separate tuning of the two modules. In addition, most practical systems already have a power control module, so a product codebook design is more easily adopted in such systems.

Although the beamforming and power control codebook design for limited feedback MISO systems is a well investigated problem, most of the existing literature deals with independent design of these codebooks. The papers that focus on beamforming often assume a fixed transmission power and investigate the optimal rank-one beamforming codebooks [5]-[9]. It has been shown that beamforming provides a bounded power gain and therefore no diversity gain ${ }^{1}$. The papers that focus on the power control, on the other hand, often assume an isotropic transmission at the transmitter and investigate the optimal power codebook [10]-[12]. It has been shown that power control can provide high diversity gains. Fig. 1 illustrates the relative gains of power control (PC) vs. beamforming $(\mathrm{BF})$.

The existing results also show that for optimal system performance, beamforming and power control modules should be used jointly, since beamforming without power control or vice versa results in a considerable power loss even with

\footnotetext{
${ }^{1}$ By diversity gain we mean the slope of the outage probability vs. the signal-to-noise ratio (SNR) curve in the logarithmic scale.
} 


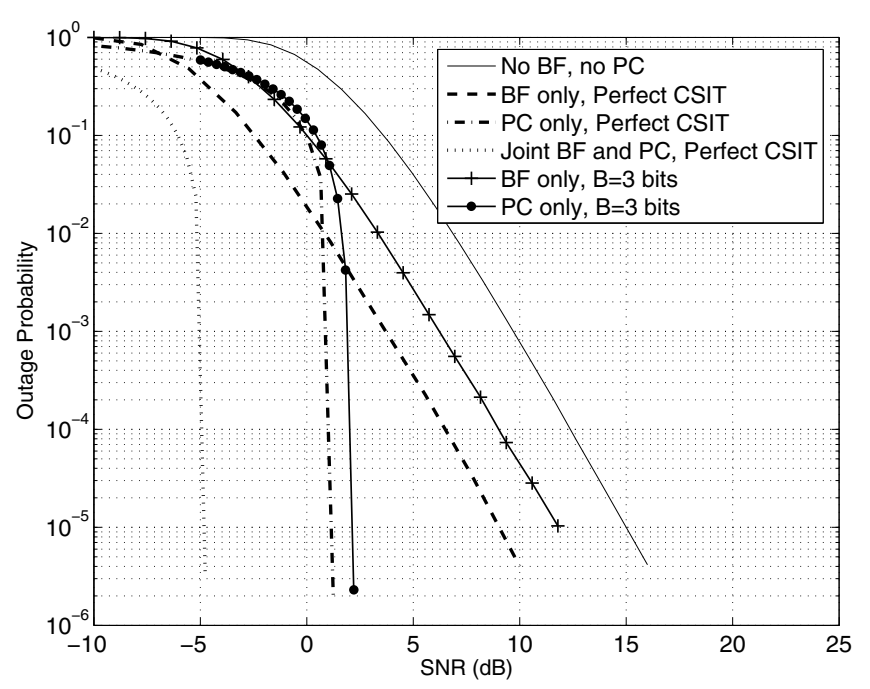

Fig. 1. Outage probability vs. SNR for MISO i.i.d Rayleigh channel with $M=4$ transmit antennas and target rate of $R=1 \mathrm{bit} / \mathrm{sec} / \mathrm{Hz}$.

infinite feedback capacity. This is evident by comparing the optimal performance, the leftmost curve in Fig. 1, with the performance of mere beamforming or power control.

The main purpose of this paper is to answer the questions of how the beamforming and power control modules should be designed jointly and how the feedback link capacity should be shared between the two modules. To this end, we formulate the optimal joint design problem as minimization of the outage probability subject to the transmit power constraint and cardinality constraints on the codebooks. In this sense, our work is related to [13], where the authors study the joint design of power, beamforming, and modulation modules of an adaptive modulation system with the objective of maximizing the average rate subject to average power and bit-error-rate constraints.

Our main contributions are as follows. We provide a general framework and a methodology for the joint optimization of beamforming and power control codebooks with fixed codebook sizes. We then proceed to show that a near-optimal performance can be achieved using a significantly simpler method in which the beamforming codebook is appropriately chosen and fixed and only the power control codebook is optimized.

Next, we address the question of how many feedback bits should be allocated to the beamforming quantization codebook and how many to power control, given a fixed feedback link capacity. We show that the answer depends on the target outage probability, but is independent of target rate. In fact, under a fixed feedback capacity, as the outage probability decreases, the power control codebook size should increase, while the beamforming codebook size should decrease. Our solution allows an optimized outage vs SNR performance for the MISO channel with limited feedback that combines the power gain of beamforming and the diversity gain of power control.

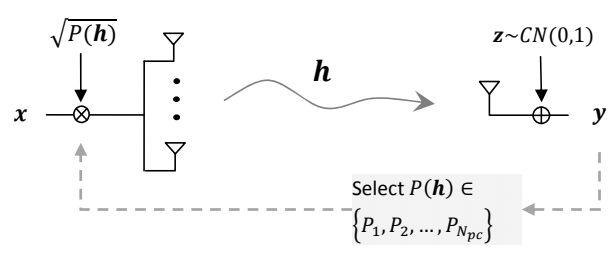

Fig. 2. Power control with limited feedback. The vector $\mathrm{x}$ is the output of an arbitrary (fixed) beamforming module.

\section{Optimal Power Control Codebook for FiXed BEAMForming MOdule}

We begin by treating a considerably simpler problem of optimizing the power control codebook for a fixed beamforming codebook. The solution to this problem is attractive numerically, as it is much simpler than a joint optimization of the two modules. As it turns out, when the fixed beamforming module is appropriately chosen, this procedure has the potential to lead to a near optimal joint codebook design.

Consider the MISO channel in Fig. 2 with $M$ transmit antennas and channel vector $\mathbf{h} \in \mathbb{C}^{M}$. The vector $\mathbf{x}$ is the output of some fixed beamforming module constrained by Trace $\left(\mathbf{Q}_{\mathbf{x} \mid \mathbf{h}}\right)=1$, where $\mathbf{Q}_{\mathbf{x} \mid \mathbf{h}}=\mathrm{E}\left[\mathbf{x} \mathbf{x}^{\dagger} \mid \mathbf{h}\right]$ is the transmit covariance matrix. The receiver knows $\mathbf{h}$ perfectly and chooses a power level, to be used by the transmitter, from the power codebook $\mathbb{P}=\left\{P_{1}, P_{2}, \cdots, P_{N_{p c}}\right\}$, where $N_{p c}$ is the number of power levels.

We want to optimize the power codebook $\mathbb{P}$ and the quantization (or power control) function $P(\mathbf{h}): \mathbb{C}^{M} \rightarrow \mathbb{P}$ such that the outage probability is minimized for a given target rate $R$ :

$$
\begin{array}{cl}
\min _{\mathbb{P}, P(\mathbf{h})} & \operatorname{Prob}\left[\log _{2}\left(1+P(\mathbf{h}) \mathbf{h}^{T} \mathbf{Q}_{\mathbf{x} \mid \mathbf{h}} \mathbf{h}^{*}\right)<R\right] \\
\text { s.t. } & \mathrm{E}[P(\mathbf{h})] \leq \mathrm{SNR} .
\end{array}
$$

Here, SNR denotes the normalized transmitter power constraint.

In order to simplify the problem, define $\gamma=\mathbf{h}^{T} \mathbf{Q}_{\mathbf{x} \mid \mathbf{h}} \mathbf{h}^{*}$ as the effective channel gain. The dependence of $\gamma$ on $\mathbf{h}$ is solely determined by the beamforming module. For example, for a limited-feedback rank-one beamforming module, we have $\mathbf{Q}_{\mathbf{x} \mid \mathbf{h}}=\mathbf{u}(\mathbf{h}) \mathbf{u}(\mathbf{h})^{\dagger}$, where the unit vector $\mathbf{u}(\mathbf{h})$ belongs to some fixed beamforming codebook. Therefore, $\gamma=\left|\mathbf{h}^{T} \mathbf{u}(\mathbf{h})\right|^{2}$.

Using the definition of $\gamma$, the problem simplifies to the following:

$$
\begin{array}{cl}
\min _{\mathbb{P}, P(\mathbf{h})} & \operatorname{Prob}[P(\gamma) \cdot \gamma<c] \\
\text { s.t. } & \mathrm{E}[P(\gamma)] \leq \mathrm{SNR},
\end{array}
$$

where $c=2^{R}-1, \gamma \geq 0$ is the quantization variable, and the optimization is over the codebook $\mathbb{P}$ and the quantization function $P(\gamma): \mathbb{R}_{+} \rightarrow \mathbb{P}$.

The structure of the problem (2) allows for explicit derivation of the optimal $P(\gamma)$ [10]. Fig. 3a shows the structure of the optimal power control function for power levels ordered in ascending order $0 \leq P_{1} \leq P_{2} \leq \cdots \leq P_{N_{p c}}$. This, in fact, can 


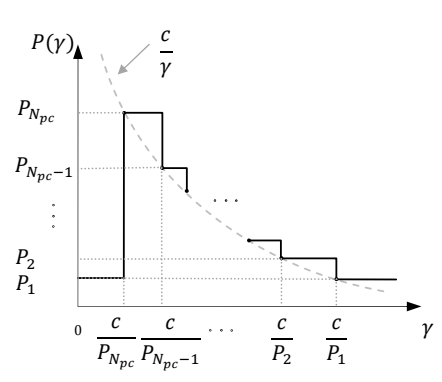

(a)

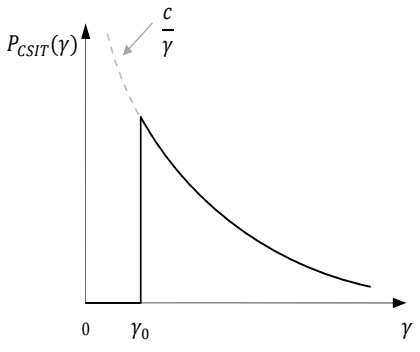

(b)
Fig. 3. (a) Optimal power control (or quantization) function with $N_{p c}$ power levels; outage only occurs when $\gamma<\frac{c}{P_{N_{p c}}}$. (b) Optimal power control with perfect CSIT: truncated channel inversion. The threshold value $\gamma_{0}$ is determined by the transmit power constraint.

be considered as a step-like approximation of the truncated channel inverting function which is the optimal power control function with perfect CSIT [14].

Now, the problem can be explicitly expressed in terms of the power levels $P_{1}, P_{2}, \cdots, P_{N_{p c}}$, since we have

$$
\begin{gathered}
\operatorname{Prob}[P(\gamma) \cdot \gamma<c]=\operatorname{Prob}\left[\gamma<\frac{c}{P_{N_{p c}}}\right]=F_{\Gamma}\left(\frac{c}{P_{N_{p c}}}\right) \\
\mathrm{E}[P(\gamma)]=P_{1} F_{\Gamma}\left(\frac{c}{P_{N_{p c}}}\right)+P_{1}\left(1-F_{\Gamma}\left(\frac{c}{P_{1}}\right)\right) \\
+\sum_{k=2}^{N_{p c}} P_{k}\left(F_{\Gamma}\left(\frac{c}{P_{k-1}}\right)-F_{\Gamma}\left(\frac{c}{P_{k}}\right)\right)
\end{gathered}
$$

where $F_{\Gamma}(\cdot)$ is the cumulative distribution function (CDF) of $\gamma$. Since a closed-form expression for $F_{\Gamma}(\cdot)$ may not be available, we interpolate the $\mathrm{CDF}$ using monotone piecewise cubic Hermite interpolation, which preserves the monotonicity of the CDF and the continuity of its first derivative [15] . Using the interpolated CDF, we can then solve (2) using the primaldual interior-point method [16]. Note that this problem is not convex and therefore we use multiple random start points to find the best among multiple local optimal solutions. For our numerical results we use 100 random starting points.

The above procedure leads to one possible joint design of power control and beamforming codebooks. For example, for Rayleigh i.i.d. channels, $\mathbf{h} \sim \mathcal{C N}\left(0, \mathbf{I}_{M}\right)$, the channel direction $\hat{\mathbf{h}}=\mathbf{h} /\|\mathbf{h}\|$ is uniformly distributed on the unit complex hypersphere. Therefore, uniform beamforming codebooks are expected to be close to optimum. It is therefore reasonable to fix the beamforming codebook as a uniform codebook and find the optimal power codebook for the fixed beamforming codebook. Note that the beamforming function should clearly be defined as $\mathbf{u}(\mathbf{h})=\arg \max _{\mathbf{u} \in \mathbb{U}_{\text {uni }}}\left|\mathbf{h}^{T} \mathbf{u}\right|^{2}$, where $\mathbb{U}_{\text {uni }}$ is the uniform codebook ${ }^{2}$. Such an approach works surprisingly well as shown in the next section. This approach is referred to as Algorithm 1 in the rest of the paper.

\footnotetext{
${ }^{2}$ By a uniform codebook, we mean a codebook that maximizes the average received SNR with fixed transmission power, $\mathrm{E}\left[\left|\mathbf{h}^{T} \mathbf{u}(\mathbf{h})\right|^{2}\right]$. This definition allows for a simple construction using Lloyd's method. Alternatively, one could use any other meaningful criterion as in [5]-[9].
}

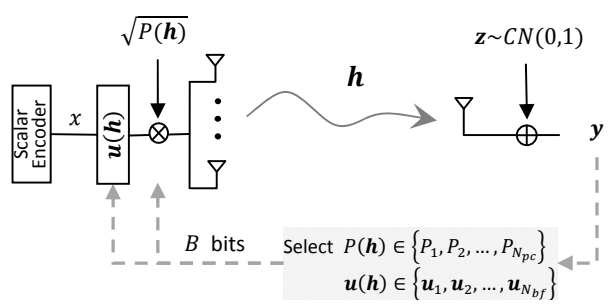

Fig. 4. Beamforming and power control with limited feedback; $N_{b f} N_{p c} \leq 2^{B}$. The scalar encoder is constrained by $\mathrm{E}\left[|x|^{2}\right]=1$.

\section{Jointly Optimal Power CONTROL AND BEAMFORMING}

We now turn to the general problem of jointly optimizing power control and beamforming codebooks for a MISO system with $M$ transmit antennas as shown in Fig. 4. The transmitter and the receiver share a beamforming codebook $\mathbb{U}=\left\{\mathbf{u}_{1}, \mathbf{u}_{2}, \cdots, \mathbf{u}_{N_{b f}}\right\}$, and a power codebook $\mathbb{P}=\left\{P_{1}, P_{2}, \cdots, P_{N_{p c}}\right\}$. For now, consider the case where $N_{b f}$ and $N_{p c}$ are fixed. The receiver chooses the beamforming vector $\mathbf{u}(\mathbf{h}) \in \mathbb{U}$ and the power level $P(\mathbf{h}) \in \mathbb{P}$ and sends the corresponding index of the $(P(\mathbf{h}), \mathbf{u}(\mathbf{h}))$ pair back to the transmitter. Clearly, we need $N_{b f} N_{p c} \leq 2^{B}$ so that the transmitter can distinguish between different $(P(\mathbf{h}), \mathbf{u}(\mathbf{h}))$ pairs.

The problem is to optimize the codebooks $\mathbb{U}, \mathbb{P}$, the beamforming function $\mathbf{u}(\mathbf{h}): \mathbb{C}^{M} \rightarrow \mathbb{U}$, and the power control function $P(\mathbf{h}): \mathbb{C}^{M} \rightarrow \mathbb{P}$, such that the outage probability is minimized:

$$
\begin{array}{cl}
\min _{\mathbb{U}, \mathbb{P}, \mathbf{u}(\mathbf{h}), P(\mathbf{h})} & \operatorname{Prob}\left[P(\mathbf{h}) \cdot\left|\mathbf{h}^{T} \mathbf{u}(\mathbf{h})\right|^{2}<c\right] \\
\text { s.t. } & \mathrm{E}[P(\mathbf{h})] \leq \mathrm{SNR},
\end{array}
$$

where $c=2^{R}-1$ and the beamforming vectors are constrained by $\left\|\mathbf{u}_{i}\right\|=1,1 \leq i \leq N_{b f}$.

The constrained optimization problem can be rephrased using a Lagrangian multiplier $\lambda$ as follows:

$$
\min _{\mathbb{U}, \mathbb{P}, \mathbf{u}(\mathbf{h}), P(\mathbf{h})} \operatorname{Prob}\left[P(\mathbf{h})\left|\mathbf{h}^{T} \mathbf{u}(\mathbf{h})\right|^{2}<c\right]+\lambda \mathrm{E}[P(\mathbf{h})] .
$$

It is possible to show that asymptotically as $B \rightarrow \infty$, the optimal outage probability vs. SNR curve is convex (in linear scale). This can be established by checking the second derivative of the outage probability expression with respect to SNR. Therefore, the optimal outage vs. SNR curve can be completely characterized by varying $\lambda$, the slope of the tangent line to the curve, and solving (6) repeatedly.

We now use the Lloyd's algorithm to find the optimal joint codebooks. First, express the objective function of (6) in form of an average distortion function $D(\mathbf{h})$ as

$$
\mathrm{E}[D(\mathbf{h})]=\mathrm{E}\left[\mathbf{1}_{c}\left(P(\mathbf{h})\left|\mathbf{h}^{T} \mathbf{u}(\mathbf{h})\right|^{2}\right)+\lambda P(\mathbf{h})\right],
$$

where the indicator function $\mathbf{1}_{c}(\cdot)$ is defined as

$$
\mathbf{1}_{c}(x)= \begin{cases}1 & \text { if } \quad x<c \\ 0 & \text { if } \quad x \geq c .\end{cases}
$$




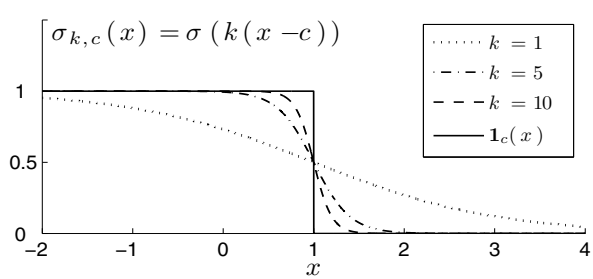

Fig. 5. $\quad \mathbf{1}_{c}(x)$ and $\sigma_{k, c}(x)$ for $c=1$ and $k=1,5,10$.

Consider $\mathbf{H}$ as a set of $S$ channel realizations. The Lloyd's algorithm iteratively updates the quantization regions and the codebooks. To update the regions, $\mathbf{u}(\mathbf{h})$ and $P(\mathbf{h})$ are chosen such that $D(\mathbf{h})$ is minimized for all $\mathbf{h} \in \mathbf{H}$ :

$$
\begin{aligned}
& \mathbf{u}(\mathbf{h})=\arg \max _{\mathbf{u} \in \mathbb{U}}\left|\mathbf{h}^{T} \mathbf{u}\right|^{2}, \\
& P(\mathbf{h})=\arg \min _{P \in \mathbb{P}} \mathbf{1}_{c}\left(P\left|\mathbf{h}^{T} \mathbf{u}(\mathbf{h})\right|^{2}\right)+\lambda P,
\end{aligned}
$$

and the regions are formed as

$$
\mathcal{H}_{i j}=\left\{\mathbf{h} \in \mathbf{H} \mid \mathbf{u}(\mathbf{h})=\mathbf{u}_{i} \text { and } P(\mathbf{h})=P_{j}\right\},
$$

where $1 \leq i \leq N_{b f}$ and $1 \leq j \leq N_{p c}$.

In order to update the codebooks, we approximate the average distortion function in (7) by the following sequence of functions:

$$
f_{k}(\mathbb{U}, \mathbb{P})=\frac{1}{S} \sum_{i=1}^{N_{b f}} \sum_{j=1}^{N_{p c}} \sum_{\mathbf{h} \in \mathcal{H}_{i j}} \sigma_{k, c}\left(P_{j}\left|\mathbf{h}^{T} \mathbf{u}_{i}\right|^{2}\right)+\lambda P_{j},
$$

where we use the approximation $\mathbf{1}_{c}(x) \approx \sigma_{k, c}(x) \stackrel{\text { def }}{=} \sigma(k(x-$ $c)$ ), where $\sigma(x)=\frac{1}{1+\exp (x)}$ is known as the sigmoid function. The parameter $k$ controls the sharpness of the approximation. Fig. 5 shows the plot of $\sigma_{k, c}(x)$ for different values of $k$. A higher $k$ gives a better approximation but also increases the magnitude of the derivative of $\sigma_{k, c}(x)$ and can make the numerical convergence more difficult. In order to minimize the average distortion function in (7) in terms of $\mathbb{U}$ and $\mathbb{P}$, we start with a small $k$ and minimize $f_{k}(\mathbb{U}, \mathbb{P})$ and use the resulting optimum as a start point for a subsequent minimization of $f_{k}(\mathbb{U}, \mathbb{P})$ with a larger $k$. This is repeated until convergence. This sequential minimization has the flavor of the interiorpoint optimization method using barrier functions where a family of approximate objective functions are optimized sequentially [16]. As $k$ increases to infinity, this process will converge to a local minimum of (7). For our numerical results we start with $k=20$ and increase it by a factor of 1.5 at each step.

The two steps of the Lloyd's algorithm are repeated until convergence. In order to fully derive the optimal outage vs. SNR curve, we vary the value of $\lambda$ and for each $\lambda$, apply Lloyd's algorithm with multiple random starts for $\mathbb{U}$ and $\mathbb{P}$. For the numerical results, 10 random start points are used for each value of $\lambda$. This procedure is referred to as Algorithm 2. Fig. 6 shows the solution sequence of this algorithm for a fixed $\lambda$ and a single start point.

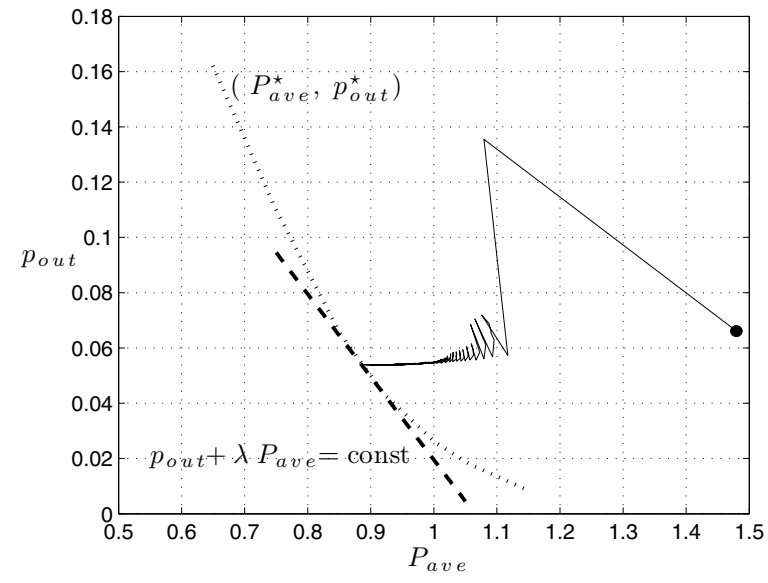

Fig. 6. The solution sequence of Algorithm 2 for $\lambda=0.3, M=3, N_{b f}=5$, $N_{p c}=3, R=1$, and $S=10^{4}$ realizations of the channel $\mathbf{h} \sim \mathcal{C N}\left(\mathbf{0}, \mathbf{I}_{M}\right)$. The filled circle shows the random start point on $\left(P_{\text {ave }}, p_{\text {out }}\right)$ plane. Both axes are in linear scale. The optimum curve $\left(P_{\text {ave }}^{\star}, p_{\text {out }}^{\star}\right)$ is generated by Algorithm 2 with different values of $\lambda$ and multiple start points for each $\lambda$.

The complexity of Algorithm 2 is quite high. But Algorithm 2 is more general and it also provides a numerical benchmark for the low-complexity fixed-beamforming-codebook approach of Algorithm 1. As shown in Fig. 7, the performance of the two algorithms are very close. Algorithm 1 in fact slightly outperforms Algorithm 2, possibly because many more random starting points are possible for Algorithm 1 due to its lower complexity. Fig. 7 also shows the performance of Algorithm 2 with the output of Algorithm 1 as its starting point - it only provides a slight improvement, suggesting that the output of Algorithm 1 is already near a local optimum for the joint design problem.

\section{Optimal Power Control and Beamforming CODEBOOK Sizes}

The final part of this paper addresses the question of given a feedback capacity of $B$ bits, how many of them should be allocated to the beamforming codebook and how many to the power control codebook. For this purpose, we use Algorithm 1 and search over all $\left(N_{b f}, N_{p c}\right)$ integer pairs that satisfy $N_{b f} N_{p c} \leq 2^{B}$ and choose the one with the best performance. The search set, however, can be considerably narrowed by considering the constraints described below.

The first constraint that we impose is $N_{b f} \geq M$. This is because with $N_{b f}<M$ we can only cover a $N_{b f}$-dimensional subspace of the $M$-dimensional complex space. This results in loss of degrees of freedom and considerably decreases the diversity gain at least for the i.i.d. Rayleigh channels.

The second constraint is based on the simple observation that once we consider a pair of beamforming and power codebook sizes $(m, n)$, we do not need to consider any other sizes $\left(m^{\prime}, n^{\prime}\right)$ with $\left(m^{\prime}, n^{\prime}\right) \leq(m, n)$ element wise, as the performance of $\left(m^{\prime}, n^{\prime}\right)$ is dominated by that of $(m, n)$. Based on this observation, we can show that for any integer $N$ and the corresponding possible codebook size pairs 


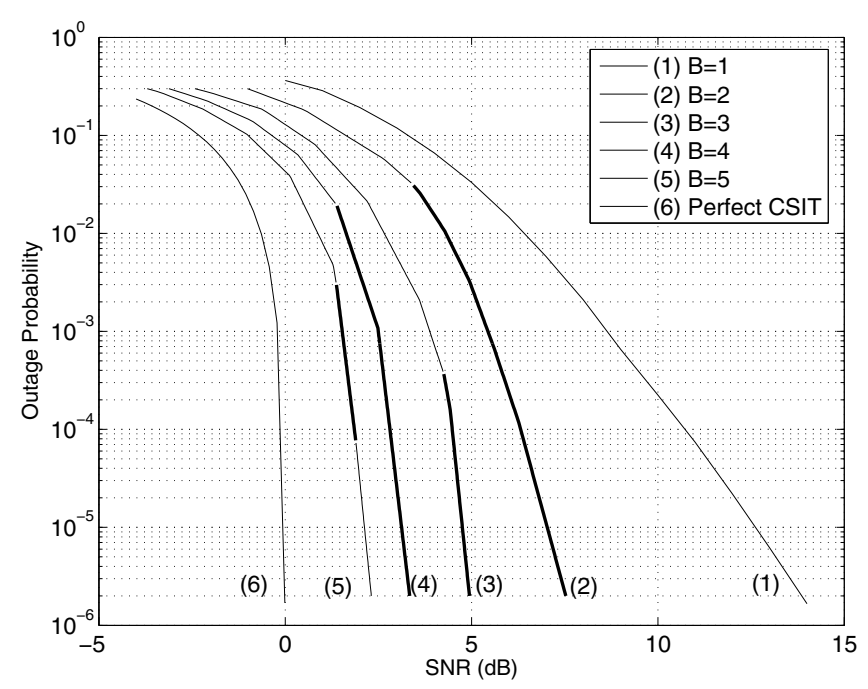

(a)

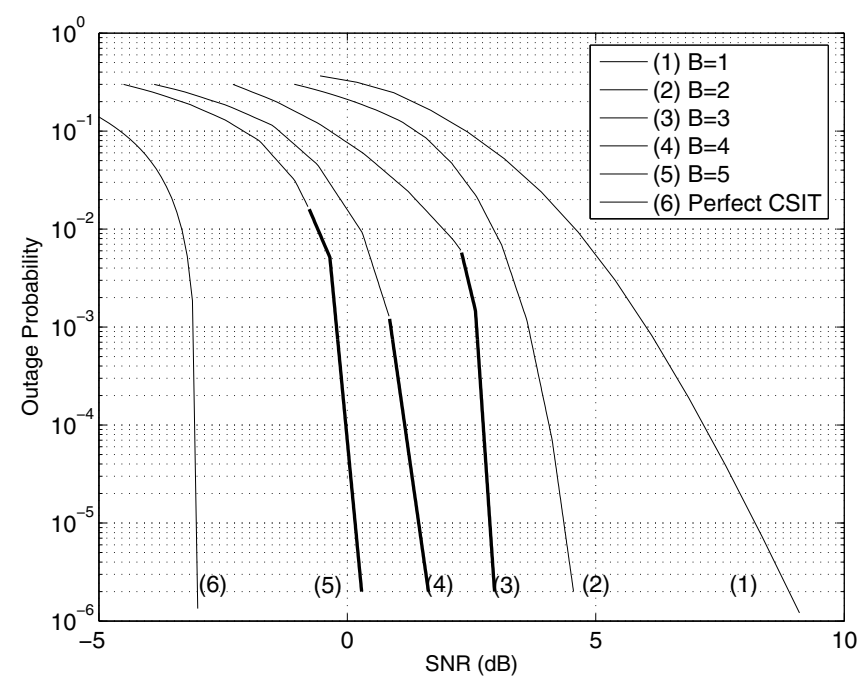

(b)

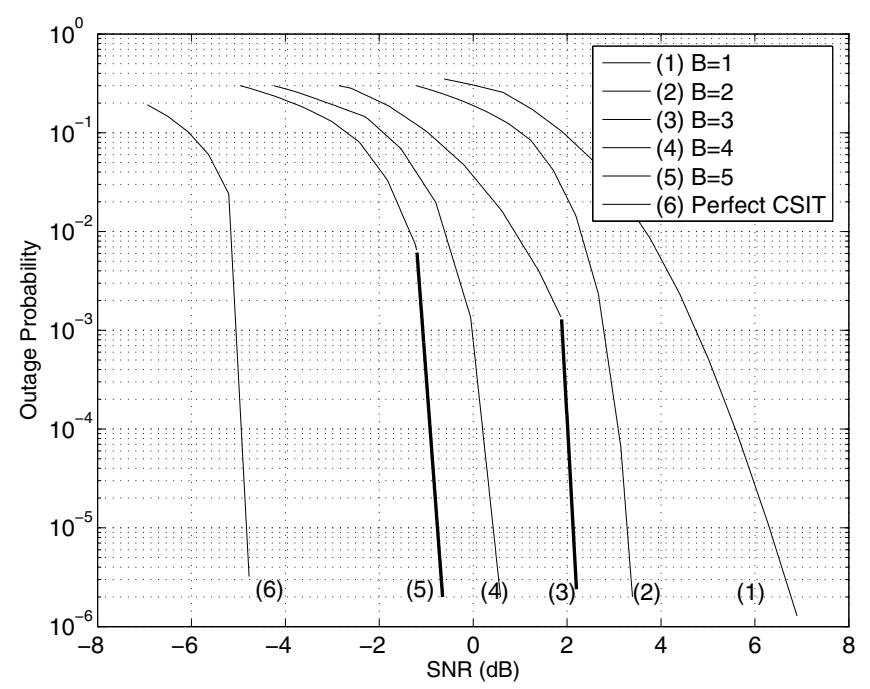

(c)

Fig. 9. Outage probability vs. SNR for joint beamforming and power control with optimal codebook sizes $(R=1 \mathrm{bit} / \mathrm{sec} / \mathrm{Hz})$; (a) $M=2$; (b) $M=3$; (c) $M=4$. feedback MISO systems. We also propose an alternative less complex method, where an appropriate beamforming codebook is chosen and only the power control is optimized, and show that its performance is close to the optimum. Given a feedback link capacity, the results are used to find the optimal codebook sizes and the corresponding optimal outage probability vs. SNR curves. It is shown that for a fixed outage probability, the optimal sizes are independent of the target rate. We further show that as the outage probability decreases, more feedback bits should be used for power control and less for beamforming. The results show that the joint design provides a combination of power gain and diversity gain, which enables it to approach the performance of the system with perfect CSIT as the feedback capacity increases - something that is not possible with either beamforming or power control alone.

\section{REFERENCES}

[1] G. Caire and S. Shamai, "On the capacity of some channels with channel state information," IEEE Trans. Inform. Theory, vol. 45, no. 6, pp. 2007 2019, Sep. 1999.

[2] V. Lau, Y. Liu, and T. Chen, "On the design of MIMO block-fading channels with feedback-link capacity constraint," IEEE Trans. Commun., vol. 52, no. 1, pp. 62-70, Jan. 2004.

[3] M. Skoglund and G. Jöngren, "On the capacity of a multiple-antenna communication link with channel side information," IEEE J. Select. Areas Commun., vol. 21, no. 3, pp. 395-405, Apr. 2003.

[4] A. Gersho and R. M. Gray, Vector Quantization and Signal Compression. Boston, MA: Kluwer, 1992.

[5] A. Narula, M. J. Lopez, M. D. Trott, and G. W. Wornell, "Efficient use of side information in multiple-antenna data transmission over fading channels," IEEE J. Sel. Areas Commun., vol. 16, no. 8, pp. 1423-1436, Oct. 1998.

[6] D. J. Love and R. W. Heath, "Grassmannian beamforming for multipleinput multiple-output wireless systems," IEEE Trans. Inform. Theory, vol. 49, no. 10, pp. 2735-2747, Oct. 2003.

[7] K. K. Mukkavilli, A. Sabharwal, E. Erkip, and B. Aazhang, "On beamforming with finite rate feedback in multiple-antenna systems," IEEE Trans. Inform. Theory, vol. 49, no. 10, pp. 2562-2579, Oct. 2003.

[8] P. Xia and G. B. Giannakis, "Design and analysis of transmitbeamforming based on limited-rate feedback," IEEE Trans. Signal Process., vol. 54, no. 5, pp. 1853-1863, May 2006.

[9] J. C. Roh and B. D. Rao, "Transmit beamforming in multiple-antenna systems with finite rate feedback: a VQ-based approach," IEEE Trans. Inform. Theory, vol. 52, no. 3, pp. 1101-1112, Mar. 2006.

[10] T. T. Kim and M. Skoglund, "Diversity-multiplexing tradeoff in MIMO channels with partial CSIT," IEEE Trans. Inform. Theory, vol. 53, no. 8, pp. 2743-2759, Aug. 2007.

[11] S. Bhashyam, A. Sabharwal, and B. Aazhang, "Feedback gain in multiple antenna systems," IEEE Trans. Commun., vol. 50, no. 5, pp. 785-798, May 2002.

[12] A. Khoshnevis and A. Sabharwal, "On the asymptotic performance of multiple antenna channels with quantized feedback." IEEE Trans. Wireless Commun., vol. 7, no. 10, pp. 3869-3877, Oct. 2008.

[13] P. Xia, S. Zhou, and G. B. Giannakis, "Multi-antenna adaptive modulation with beamforming based on bandwidth-constrained feedback," IEEE Trans. Commun., vol. 53, no. 3, pp. 526-536, Mar. 2005

[14] G. Caire, G. Taricco, and E. Biglieri, "Optimum power control over fading channels," IEEE Trans. Inform. Theory, vol. 45, no. 5, pp. 14681489, Jul. 1999.

[15] F. N. Fritsch and R. E. Carlson, "Monotone piecewise cubic interpolation," SIAM J. Numer. Anal., vol. 17, no. 2, pp. 238-246, Apr. 1980.

[16] S. Boyd and L. Vandenberghe, Convex Optimization. Cambridge University Press, 2004. 